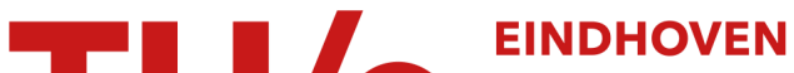 UNIVERSITY OF TECHNOLOGY
}

\section{Edge contribution to forward scattering by spheres}

\section{Citation for published version (APA):}

Bosch, van den, H. F. M., Ptasinski, K. J., \& Kerkhof, P. J. A. M. (1996). Edge contribution to forward scattering by spheres. Applied Optics, 35(13), 2285-2291. https://doi.org/10.1364/AO.35.002285

DOI:

10.1364/AO.35.002285

Document status and date:

Published: 01/01/1996

\section{Document Version:}

Publisher's PDF, also known as Version of Record (includes final page, issue and volume numbers)

\section{Please check the document version of this publication:}

- A submitted manuscript is the version of the article upon submission and before peer-review. There can be important differences between the submitted version and the official published version of record. People interested in the research are advised to contact the author for the final version of the publication, or visit the $\mathrm{DOI}$ to the publisher's website.

- The final author version and the galley proof are versions of the publication after peer review.

- The final published version features the final layout of the paper including the volume, issue and page numbers.

Link to publication

\section{General rights}

Copyright and moral rights for the publications made accessible in the public portal are retained by the authors and/or other copyright owners and it is a condition of accessing publications that users recognise and abide by the legal requirements associated with these rights.

- Users may download and print one copy of any publication from the public portal for the purpose of private study or research.

- You may not further distribute the material or use it for any profit-making activity or commercial gain

- You may freely distribute the URL identifying the publication in the public portal.

If the publication is distributed under the terms of Article 25fa of the Dutch Copyright Act, indicated by the "Taverne" license above, please follow below link for the End User Agreement:

www.tue.nl/taverne

Take down policy

If you believe that this document breaches copyright please contact us at:

openaccess@tue.nl

providing details and we will investigate your claim. 


\title{
Edge contribution to forward scattering by spheres
}

\author{
H. F. M. van den Bosch, K. J. Ptasinski, and P. J. A. M. Kerkhof
}

\begin{abstract}
Edge functions $T 1$ and $T 2$, which describe the polarization-dependent edge contribution to forward scattering by spheres, are derived from the exact Mie solution. All the relative refractive indices and the $64<x<2048$ size parameter range are considered. The edge functions significantly improve the approximation methods that can be used to calculate forward-scattering patterns. For $m$ close to 1, an asymptotic approximation is used. Otherwise, the familiar geometrical optics approximation and the similar physical optics approximation for glory rays are used. Both geometrical and physical optics equations can be deduced from the above-mentioned asymptotic approximation.

Key words: Forward light scattering, sphere, Mie, geometrical optics, physical optics, anomalous diffraction, edge function. ๔ 1996 Optical Society of America
\end{abstract}

\section{Introduction}

A widely used method for determining particle sizes is measurement of the forward-scattering pattern of a dilute suspension of particles over an integer number of detectors. If no multiple scattering occurs, the measured scattering vector $\overline{\mathbf{b}}$ is the sum of scattering by all the particles of different sizes:

$$
\overline{\overline{\mathscr{A}}} \cdot \bar{x}=\overline{\mathbf{b}},
$$

where $\bar{x}$ is a discrete size distribution and $\overline{\overline{\mathscr{C}}}$ is a scattering matrix whose columns are the scattering vectors of a number of size classes. The range of sizes that can be distinguished depends on the combination of wavelength and angles for which the scattering pattern is measured.

Calculation of scattering matrix $\overline{\overline{\mathscr{C}}}$ requires calculation of the forward-scattering patterns of particles in the considered size range. For spherical particles, with any relative refractive index $m=$ $n_{\text {sphere }} / n_{\text {medium }}$ and size parameter $x=2 \pi a / \lambda$, calculation of the exact solution according to $\mathrm{Mie}^{1,2}$ is possible, but this requires a long calculation time for large values of $x$.

The authors are with the Department of Chemical Engineering, Eindhoven University of Technology, P.O. Box 513, 5600 MB Eindhoven, The Netherlands.

Received 11 September 1995; revised manuscript received 18 December 1995.

0003-6935/96/132285-07\$10.00/0

(1) 1996 Optical Society of America
For $x$ large and $m$ not too close to 1 , it is possible for one to calculate the scattering pattern of a sphere by calculating separately the contributions from incident and nonincident rays. The incomplete wave front of the nonincident rays gives a Fraunhofer diffraction pattern. The energy of an incident ray is divided among several outgoing rays $\mathrm{p}$ (see Fig. 1), giving several contributions to the scattering pattern that can be calculated using ray or geometrical optics.

Glory rays, i.e., nonaxial rays that are scattered in the forward or backward direction, have infinite intensity according to the geometrical optics (GO) approximation. Langley and co-workers ${ }^{3,4}$ detail a physical optics (PO) approximation, using both ray optics and diffraction theory, that is valid for these rays. Both $\mathrm{GO}$ and $\mathrm{PO}$ equations can be deduced from the exact solution with an asymptotic approximation presented by van de Hulst. ${ }^{5}$

This asymptotic approximation cannot be used for rays incident on the edge of the sphere, i.e., for small values of $\tau$. These rays are partially reflected rays $(p=0)$ close to the forward direction and diffract into the shadow region behind the sphere just like the rays that miss the particle and cause the Fraunhofer diffraction. The edge domain for which reflected rays are significantly diffracted depends on the size parameter and is given by $\tau<x^{-1 / 3}$ (van de Hulst ${ }^{6}$ ), i.e., for large values of $x$ the edge contribution can be neglected.

We show that the difference between the exact Mie solution and the approximation for forward-scattering patterns, for moderate values of $x(64-2048)$ and 


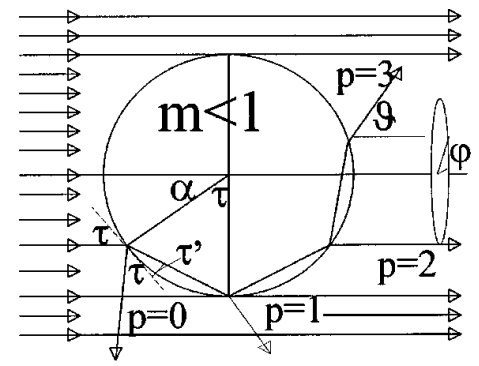

Fig. 1. Separation of wave front into incident and nonincident rays for a sphere with relative refractive index $m<1$. The hole in the wave front causes a Fraunhofer diffraction pattern. The incident rays are partly reflected and partly refracted. Several outgoing rays are denoted by $p=0,1,2,3$.

averaged over a size range $\Delta x$, results from the edge domain. This edge contribution can be expressed by two edge functions, ${ }^{6} T 1$ and $T 2$, that can be represented by several polynomials of $x$ and $m$. The averaged Mie solution can now be reconstructed by adding the approximation and the edge contribution.

There are other approximate calculation methods for light scattering that also have separate terms for the edge contribution. Nussenzveig ${ }^{7-9}$ presented the complex angular momentum (CAM) theory that gives a physical explanation for all the features of the exact Mie solution. The CAM theory allows approximate expressions that are valid for $x^{1 / 3} \gg 1$ and $\left.|m-1|\right|^{1 / 2} x^{1 / 3} \gg 1$. An expression for the extinction efficiency ${ }^{10}$ is compared with our result.

Chen ${ }^{11,12}$ generalized the eikonal approximation (valid for scalar waves and $m$ near 1) with two parameters and adapted these parameters to fit the approximation to the exact solution in the forward direction. The maximum size parameter for which a scattering pattern $0<\vartheta<30^{\circ}$ is presented is $x=$ 30 together with $m=1.33$. The GO approximation with an edge contribution is a better approximation for this case, possibly because the difference for both polarizations at the edge is not accounted for in a generalized eikonal approximation. Calculation times for both methods are not compared.

\section{Asymptotic Approximation}

The asymptotic approximation that was used by van de Hulst ${ }^{5}$ to demonstrate the correspondence of the exact solution and the GO approximation is used to derive an asymptotic formula $(\mathrm{AF})$ that can be used to approximate the forward-scattering pattern for $m \approx$ 1. Sharma ${ }^{13}$ used the same approximation to explain the large range of $m$ values for which the anomalous diffraction approximation gives good results for extinction efficiency $Q_{\text {ext }}=4 S(0) / x^{2}$. The exact solution is given by ${ }^{1}$

$$
S 1(x, \vartheta)=\sum_{n=1}^{\infty} \frac{2 n+1}{n(n+1)}\left[a_{n} \pi_{n}(\cos \vartheta)+b_{n} \tau_{n}(\cos \vartheta)\right] .
$$

The expression for $S 2$ can be obtained from $S 1$ by interchanging $a_{n}$ and $b_{n}$. Both scattering coeffi- cients,

$$
a_{n}=\frac{1-\exp \left(-2 i \alpha_{n}\right)}{2}, \quad b_{n}=\frac{1-\exp \left(-2 i \beta_{n}\right)}{2},
$$

contain the term $1 / 2$ that corresponds with Fraunhofer diffraction and is omitted from further consideration. The remaining terms depend on size parameter $x$ and the relative refractive index $m$. For large $x$ and excluding the edge domain, the following asymptotic approximation can be used ${ }^{5}$ :

$$
\begin{aligned}
-\exp \left(-2 i \alpha_{n}\right) & \approx u_{0} \frac{u_{i}+r 2}{1+u_{i} r 2} \\
& =u_{0}\left[r 2+\left(1-r 2^{2}\right) \sum_{p=1}^{\infty} u_{i}^{p}(-r 2)^{p-1}\right],
\end{aligned}
$$

where reflection coefficient $r 2=\tan \left(\tau-\tau^{\prime}\right) / \tan \left(\tau+\tau^{\prime}\right)$. Angles $\tau$ and $\tau^{\prime}$ are defined by

$$
x \cos \tau=x^{\prime} \cos \tau^{\prime}=n+1 / 2,
$$

where $x^{\prime}=m x$. The separate terms on the righthand side of approximation (3) correspond to rays $p=0,1,2$, etc. (Fig. 1). A similar approximation is allowed for $-\exp \left(-2 i \beta_{n}\right)$ with $r 2$ replaced by $r 1=\sin \left(\tau-\tau^{\prime}\right) / \sin \left(\tau+\tau^{\prime}\right)$. The function $u_{0}=-i$ $\exp (-2 i x f)$ with $f=\sin \tau-\tau \cos \tau$ contains outside values, and $u_{i}=-i \exp \left(2 i x^{\prime} f^{\prime}\right)$ with $f^{\prime}=\sin \tau^{\prime}-$ $\tau^{\prime} \cos \tau^{\prime}$ contains inside values for the angle and size parameter. The notation used here deviates from the notation used by van de Hulst insofar as $\exp (-i \omega t)$ instead of $\exp (i \omega t)$ was used to represent time dependence.

van de Hulst ${ }^{5}$ also replaced the angular functions $\pi_{n}$ and $\tau_{n}$ in Eq. (1) with asymptotic approximations that are valid for all scattering angles $\vartheta$ except $\vartheta \approx 0$ and $\vartheta \approx \pi$. After several additional steps, neglecting $\pi_{n}$ and the stationary phase approximation, the GO equations were found. The replacement of functions $\pi_{n}$ and $\tau_{n}$ by asymptotic approximations that are valid near the forward or backward direction for large $n$, i.e., excluding axial rays, has already been suggested by van de Hulst:

$$
\begin{aligned}
& \pi_{n}(\cos \vartheta) \approx n(n+1) \frac{J_{1}(u)}{u} s^{n-1}, \\
& \tau_{n}(\cos \vartheta) \approx n(n+1) J_{1}{ }^{\prime}(u) s^{n},
\end{aligned}
$$

where $u=(n+1 / 2) \vartheta$ and $s=1$ for the forward direction whereas $u=(n+1 / 2)(\pi-\vartheta)$ and $s=-1$ for the backward direction. The angular functions can also be written using $J_{1}(u) / u=\left(J_{0}+J_{2}\right) / 2$ and $J_{1}{ }^{\prime}=$ $\left(J_{0}-J_{2}\right) / 2$, where $J_{0}, J_{1}$, and $J_{2}$ are the first three integer Bessel functions of the first kind.

The forward-scattering amplitude, excluding Fraunhofer diffraction and reflection terms and using the average reflection coefficient $r=(r 1+r 2) / 2$, 
can now be approximated by

$$
\begin{aligned}
\frac{S 1(x, \vartheta)}{x^{2}}= & \int_{\tau_{\text {low }}}^{\pi / 2} u_{0}\left(\frac{u_{i}+r}{1+u_{i} r}-r\right) \\
& \times J_{0}(x \vartheta \cos \tau) \cos \tau \sin \tau \mathrm{d} \tau,
\end{aligned}
$$

where the summation of Eq. (1) is replaced by an integral over $\mathrm{d} n=d(x \cos \tau)=x \sin \tau \mathrm{d} \tau$. This replacement is allowed if the integrand is a slowly varying function of $\tau$. For $m<1$ the lower limit of the integral in Eq. (6) is therefore taken as $\tau_{\text {low }}=$ $\tau_{\text {crit }}\left(\cos \tau_{\text {crit }}=m\right)$, whereas for $m>1, \tau_{\text {low }}=0$. Equation (6) with $r=0, u_{0} u_{i}=-\exp (i \rho \sin \tau), \rho=$ $2 x(m-1)$, and $\tau_{\text {low }}=0$ gives the anomalous diffraction approximation (without Fraunhofer diffraction).

The advantage of this asymptotic formula over the exact Mie solution is the following. Regardless of the value of $m$, the Mie algorithm makes a summation over a little more than $x$ terms. The integrand in Eq. (6) is for $m$ near 1, a slowly varying function of $\tau$, and for sizing systems using forward scattering the integral can be determined numerically accurately with $\mathrm{O}(1) x^{2 / 3}$ terms.

Whereas the cumulative integral of the anomalous diffraction approximation for $\vartheta=0$ in the complex domain gives a smooth spiral, the corresponding cumulative AF result, i.e., Eq. (6), for $\vartheta=0$ gives approximately the same spiral with an oscillating curvature.

\section{Glory Rays}

We derive an equation for glory rays, i.e., nonaxial rays that are scattered in the forward or backward direction (e.g., $p=2$ in Fig. 1), using the approximations outlined in Section 2 . The result matches the PO approximation by Langley and co-workers ${ }^{3,4}$ who also considered the rainbow-enhanced glory that we do not consider here.

The derivation that we present is similar to that of the GO equations by van de Hulst. ${ }^{5}$ Substituting the approximations [Eq. (2) and approximations (3)(5)] in Section 2 for one value of $p>1,\left(1-r 2^{2}\right)(-r 2)^{p-1}$ $=\epsilon 2_{p}$ and $\left(1-r 1^{2}\right)(-r 1)^{p-1}=\epsilon 1_{p}$ in Eq. (1), leads to

$$
\begin{aligned}
S 1_{p}(x, \vartheta)= & \sum_{n=1}^{\infty}\left(n+\frac{1}{2}\right)\left[\epsilon 2_{p} \frac{J_{1}(u)}{u s}+\epsilon 1_{p} J_{1}^{\prime}(u)\right] \\
& \times u_{0} u_{i}^{p} s^{n} .
\end{aligned}
$$

The last three factors of Eq. (7) can be rewritten as

$$
\begin{aligned}
u_{0} u_{i}^{p} s^{n} & =\exp \left(-i G_{n}\right) \\
G_{n} & =2\left(x f-p x^{\prime} f^{\prime}\right)+(p+1) \frac{\pi}{2}-L \pi n .
\end{aligned}
$$

In the forward direction $L=0$ and in the backward direction $L=1$. The complex terms nearly cancel one another except near the value of $n=n_{0}$, where $G_{n+1}-G_{n}+k 2 \pi=0$ is nearly satisfied. Integer $k$ can be omitted if $L$ is allowed to assume any even value in the forward direction and any uneven value in the backward direction. Replacing the difference with the derivative

$$
\frac{d}{\mathrm{~d} n}=\frac{1}{x} \frac{d}{d \cos \tau}=\frac{-1}{x \sin \tau} \frac{d}{\mathrm{~d} \tau}
$$

leads to

$$
G_{n}{ }^{\prime}=-2 \tau+2 p \tau^{\prime}-L \pi=0
$$

for a forward or backward directed ray. This condition can be used to determine the values of $n_{0}, \tau$, and $\tau^{\prime}$. Multiplying this equation with $n_{0}+1 / 2=$ $x \cos \tau=x^{\prime} \cos \tau^{\prime}$ and subtracting the product from $G_{n}$ give the following expression for the phase delay of such a ray:

$$
G_{n o}=2 x \sin \tau-2 p x^{\prime} \sin \tau^{\prime}+(p+1+L) \pi / 2 .
$$

For the summation around $n_{0}$ the first two factors of Eq. (7) are quasi-constant. Replacing the summation by an infinite integral and $G_{n}$ by a second-order approximation leads to the Fresnel integral

$$
\begin{aligned}
\int \exp \left[-i G_{n o}-\right. & \left.i \frac{1}{2}\left(n-n_{o}\right)^{2} G_{n o}{ }^{\prime \prime}\right] \mathrm{d} n \\
& =\left(\frac{2 \pi}{\mid G_{n o}{ }^{\prime \prime}}\right)^{1 / 2} \exp \left[-i G_{n o}-i(\pi / 4) s_{A}\right]
\end{aligned}
$$

where

$$
G_{n o}{ }^{\prime \prime}=\frac{-A}{x \cos \tau}, \quad A=\frac{2 p}{\tan \tau^{\prime}}-\frac{2}{\tan \tau},
$$

and $s_{A}$ represents the sign of $A$. The resulting amplitude function is given by

$$
\begin{aligned}
S 1_{p}(x, \vartheta)= & (x \cos \tau)^{3 / 2}\left(\frac{2 \pi}{|A|}\right)^{1 / 2}\left[\epsilon 2_{p} \frac{J_{1}(u)}{u s}+\epsilon 1_{p} J_{1}{ }^{\prime}(u)\right] \\
& \times \exp \left[-i G_{n o}-i(\pi / 4) s_{A}\right],
\end{aligned}
$$

where $u=x \vartheta \cos \tau$ or $u=x(\pi-\vartheta) \cos \tau$ for the forward or backward direction, respectively, and $\tau$ is determined by Eq. (9). One can obtain the expression for $S 2$ by interchanging $\epsilon 1_{p}$ and $\epsilon 2_{p}$. The expression in brackets corresponds to a virtual ring source with diameter $u / \vartheta=x \cos \tau$ as photographed by Langley and co-workers ${ }^{4,5}$ who found a corresponding equation using ray optics and diffraction theory (PO approximation). Near the rainbow condition $(A=0)$ a third-order approximation of $G_{n}$ is required, which we do not discuss in this paper.

\section{Edge Functions}

It is evident from Fig. 2 that two different amplitude functions, $S 1(\vartheta)$ and $S 2(\vartheta)$, are required to give a description of the far-field scattering behavior of a sphere $(x, m)$ placed in a linearly polarized flat electromagnetic wave. For a plane at any azimuth angle 
$\varphi$, decomposition is necessary to find the amplitude of the scattered wave. Amplitude function $S 1$ depends on reflection (and transmission) with the electric field normal to the plane of incidence, i.e., on reflection coefficient $r 1$, whereas $S 2$ depends on reflection with the electric field in the plane of incidence, i.e., coefficient, $r 2$. This is correct for large angle scattering. Close to the forward and backward directions, i.e., $\vartheta \approx 0$ or $\pi$, rays that emerge from all azimuth angles $\varphi$ contribute significantly by way of diffraction to both amplitude functions that depend on both reflection coefficients, as is the case for glory rays [Eq. (12)].

The edge rays are reflected in the forward direction. Their contributions $S 1_{\text {edg }}$ and $S 2_{\text {edg }}$ to the total amplitude functions are assumed to be diffraction patterns that radiate from a circle around the sphere (dotted circle in Fig. 2). The width of the relevant wave front is neglected. The actual relevant edge domain is given by a specific value of angle $\tau$ (Fig. 1) that depends on size parameter $x: \tau_{\text {edg }}=x^{-1 / 3}$. The width of the relevant part of the incident wave front is thus given by $x\left(1-\cos \tau_{\text {edg }}\right) \approx x^{1 / 3} / 2$, which, for large values of $x$, is small compared with the circumference of the circle. Since reflection coefficients $r 1$ and $r 2$ for both possible polarizations are different, two different edge functions, $T 1$ and $T 2$, are needed to describe the contribution from any azimuth angle $\varphi$.

Decomposition of the original polarization direction into two components, normal and parallel to the scattering plane, and decomposition of the resulting amplitudes after reflection to the original polarization direction along with integration over the mentioned circle at the edge leads to the following equations for the amplitude functions:

$$
\begin{aligned}
& \frac{S 1_{\mathrm{edg}}(x, z)}{2 x}=T 1(x) J_{1}{ }^{\prime}(z)+T 2(x) \frac{J_{1}(z)}{z}, \\
& \frac{S 2_{\mathrm{edg}}(x, z)}{2 x}=T 2(x) J_{1}{ }^{\prime}(z)+T 1(x) \frac{J_{1}(z)}{z},
\end{aligned}
$$

where $z=x \sin \vartheta \approx x \vartheta$ and the edge functions are assumed independent of scattering angle $\vartheta$.

Assuming that the edge contributions $S 1_{\text {edg }}$ and $S 2_{\text {edg }}$ to the total scattering amplitudes are known, both edge functions follow directly from the first null point in $J_{1}{ }^{\prime}\left(z_{a}\right)=0, z_{a}=1.84 \ldots$ (hereafter referred to

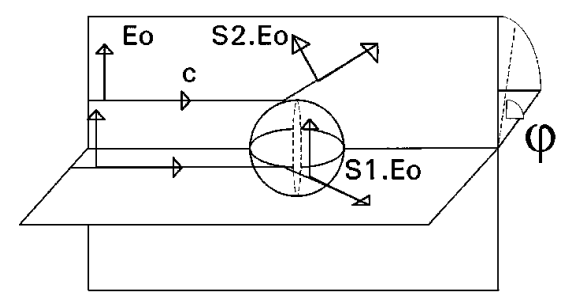

Fig. 2. Linearly polarized light, after scattering by a sphere, is still linearly polarized in the planes normal and parallel to the polarization direction. as method a),

$$
T 1_{a}(x)=\frac{S 2_{\text {edg }}\left(x, z_{a}\right)}{2 x J_{1}\left(z_{a}\right) / z_{a}}, \quad T 2_{a}(x)=\frac{S 1_{\text {edg }}\left(x, z_{a}\right)}{2 x J_{1}\left(z_{a}\right) / z_{a}},
$$

and also from the first null point in $J_{1}\left(z_{b}\right)=0, z_{b}=$ 3.83 ... (hereafter referred to as method b),

$$
T 1_{b}(x)=\frac{S 1_{\text {edg }}\left(x, z_{b}\right)}{2 x_{1_{1}}{ }^{\prime}\left(z_{b}\right)}, \quad T 2_{b}(x)=\frac{S 2_{\text {edg }}\left(x, z_{b}\right)}{2 x_{J_{1}}{ }^{\prime}\left(z_{b}\right)} .
$$

If the considered amplitude functions $S 1$ and $S 2$ indeed result from the circle at the edge of the sphere, then both methods should give identical edge functions: $T 1_{a}=T 1_{b}$ and $T 2_{a}=T 2_{b}$. Because of the actual ratio of the width of the relevant wave front to the diameter of the circle around the sphere given by $x^{1 / 3} / x=x^{-2 / 3}$, the differences $T 1_{a}-T 1_{b}$ and $T 2_{a}-T 2_{b}$ are smaller for larger values of $x$. Using $J_{0}(0)=1$ and $J_{2}(0)=0$ the following relations must also be true:

$$
\begin{aligned}
S 1_{\text {edg }}(x, 0) & =S 2_{\text {edg }}(x, 0) \approx x\left(T 1_{a}+T 2_{a}\right) \\
& \approx x\left(T 1_{b}+T 2_{b}\right) .
\end{aligned}
$$

One can calculate amplitude functions $S 1_{\text {edg }}$ and $S 2_{\text {edg }}$ by subtracting from the exact Mie solution different approximations in different ranges of the complex relative refractive index $m$.

\section{Absorbing Sphere}

The simplest case for determining the edge functions according to the information in Section 4 is the value of $m$ with a large imaginary part so that all $p>0$ contributions are absorbed and only the Fraunhofer diffraction and edge contribution are important for the forward-scattering pattern: $S_{\text {edg }}=S_{\mathrm{Mie}}-S_{\mathrm{Frh}}$. For such a subtraction we use the short notation of Mie-Frh. Figure 3 shows the resulting real part of edge functions $T 1$ and $T 2$ according to method $\mathrm{b}$ for $64<x<2048,0.1<m<100$, and $\operatorname{Im}[m]=\operatorname{Re}[m]$. Figure 4 shows that the differences $T 1_{a}-T 1_{b}$ and $T 2_{a}-T 2_{b}$ between the results from method a and method $\mathrm{b}$ for $x=64$ are an order of magnitude smaller than for $T 1_{b}$ and $T 2_{b}$, whereas for larger values of $x$ the difference decreases as $x^{-2 / 3}$. This result shows that it is indeed possible to deduce the edge functions from the exact Mie solution and including these edge functions in the approximation will decrease the approximation error significantly.

From a comparison of differences $S 1(x, 0)-$ $x\left(T 1_{a}+T 2_{a}\right)$ and $S 1(x, 0)-x\left(T 1_{b}+T 2_{b}\right)$ it was found that the use of method $b$ gives a slightly better approximation in the exact forward direction than does method a.

\section{Transparent Sphere}

The largest contribution to forward scattering besides the Fraunhofer diffraction and the edge reflection for real values of $m$ is the twice refracted $p=1$ ray, leading to the definition $S_{\mathrm{GO}}=S_{\mathrm{Frh}}+S_{p=1}$, 

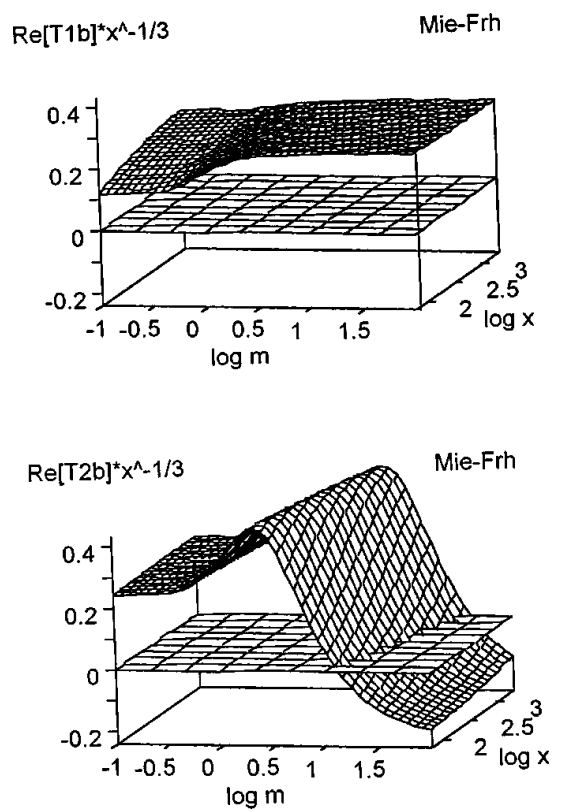

Fig. 3. Real part of edge functions $T 1$ and $T 2$ multiplied by $x^{-1 / 3}$, calculated using method b for $0.1<m<100, \operatorname{Im}[m]=\operatorname{Re}[m]$, and $64<x<2048$.

where $S_{p=1}$ was calculated according to GO. One can diminish the $p>1$ contributions to $S_{\text {Mie }}$ by averaging $S_{\mathrm{Mie}}-S_{\mathrm{GO}}$ over a size range of $\Delta x \approx x / 8$, leading to the edge contribution $S_{\text {edg }}=\overline{S_{\mathrm{Mie}}-S_{\mathrm{GO}}}$. This is true for the largest part of the $m-x$ range shown in Fig. 5 but not for the area around $m=1$ bounded by $\mu=-16$ and $\rho=2 x(m-1)=51$. The $\mu$ parameter is defined here as $\mu=(m-1) x^{2 / 3} \approx$ $\left(\tau_{\text {crit }} / \tau_{\text {edg }}\right)^{2}$ and depends on the position of the critical angle in the edge domain.
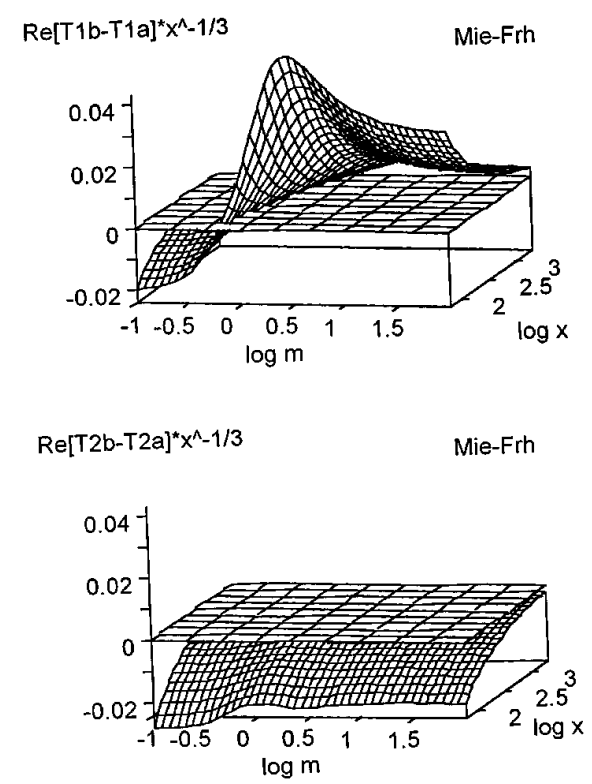

Fig. 4. Difference between the edge functions in Fig. 3 and the corresponding edge functions that were calculated using method a.

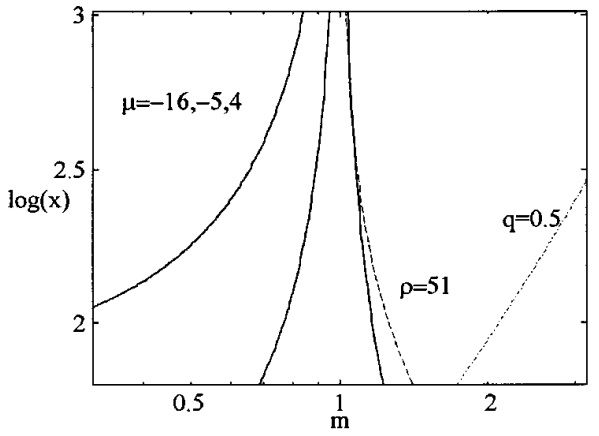

Fig. 5. The $m-x$ area for $0.3<m<3$ and $64<x<1024$, divided into areas bounded by indicated conditions for $\mu, \rho$, and $q$.

For $-16<\mu<-5$ the glory rays of the type discussed in Section 3 are not sufficiently diminished by averaging and are included in the definition $S_{\mathrm{PO}}=$ $S_{\mathrm{GO}}+S_{p=2,9}$, where the second term is the sum of the glory contributions according to the PO approximation for $p=2$ to $p=9$ with $L=0$. In this range the edge contribution is given by $S_{\mathrm{edg}}=\overline{S_{\mathrm{Mie}}-S_{\mathrm{PO}}}$.

In the remaining range for $-5<\mu$ and $\rho<51$ the difference between the exact solution $S_{\mathrm{Mie}}$ and the asymptotic approximation of Eq. (6) including the Fraunhofer diffraction, referred to as $S_{\mathrm{AF}}$, leads to the edge contribution $S_{\text {edg }}=\overline{S_{\mathrm{Mie}}-S_{\mathrm{AF}}}$. For the $-4<\mu<4$ range Fig. 6 shows the resulting edge function $T 1_{b}$ [Eq. (16)] and the difference $T 1_{a}-T 1_{b}$, which is much smaller than $T 1_{b}$, indicating that in
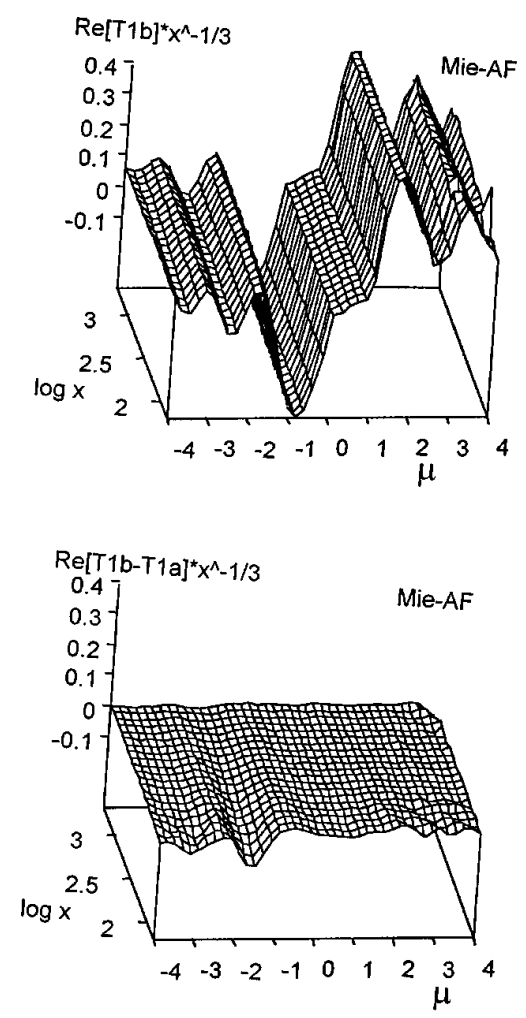

Fig. 6. Real part of edge function $T 1$ multiplied by $x^{-1 / 3}$, calculated according to method $b$ (upper figure) for $-4<\mu<4$ and $64<x<2048$, and the difference in the corresponding function that was calculated according to method a (lower figure).

1 May 1996 / Vol. 35, No. 13 / APPLIED OPTICS 
this range $T 1_{b}$ may indeed be interpreted as an edge function. The edge functions in this range, multiplied by $x^{-1 / 3}$, are a function of a parameter $\mu$ and marginally a function of $x$. Directly below $\mu=0$ the Goos-Hanchen effect ${ }^{9,14}$ probably causes the deep dip in the edge function, whereas the rest of the fluctuations can be interpreted as the results of continuous diffraction interaction between incident, reflected, and inside waves in the edge domain.

For $|\rho|<0.01$ we used the Rayleigh-Gans ${ }^{15}$ approximation. In our figures only the $m=1$ line corresponds to this case. For $m>1$ the reflection coefficient $r 2$ changes sign at the Brewster angle $\tau_{\text {Brw }}$, where $\tau+\tau^{\prime}=\pi / 2$. Parameter $q$ as defined here gives the inverse of the relative position of the Brewster angle in the edge domain: $q=$ $\left(1+m^{2}\right)^{1 / 2} x^{-1 / 3}=\tau_{\text {edg }} / \sin \tau_{\text {Brw }}$. Figure 7 shows the functions $T 1_{b}$ and $T 2_{b}$ as functions of parameter $q$ calculated for $S_{\text {edg }}=\overline{S_{\mathrm{Mie}}-S_{\mathrm{GO}}}$. The remaining ripple in Fig. 7 is due to rays with $p>1$ and can be diminished with a better averaging method. Near $q=0.1$ and $x=64$, refractive index $m$ would be less than 1 based on the definition of $q$, so the edge function is simply set to 0 . The flat region was calculated for a refractive index with a small imaginary component $\left(\operatorname{Im}[m] / \operatorname{Re}[m]<10^{-4}\right)$ without averaging.

For $64<x<1024$ we determined different polynomials of the functions $T 1_{b} x^{-1 / 3}$ and $T 2_{b} x^{-1 / 3}$ as functions of $x$ and one of the real parameters $m, \mu$, or $q$. It does not seem to be difficult to extend the polynomials to complex values of $m$.

The entire forward-scattering pattern within the penumbra region ${ }^{7}$ can be calculated by adding the
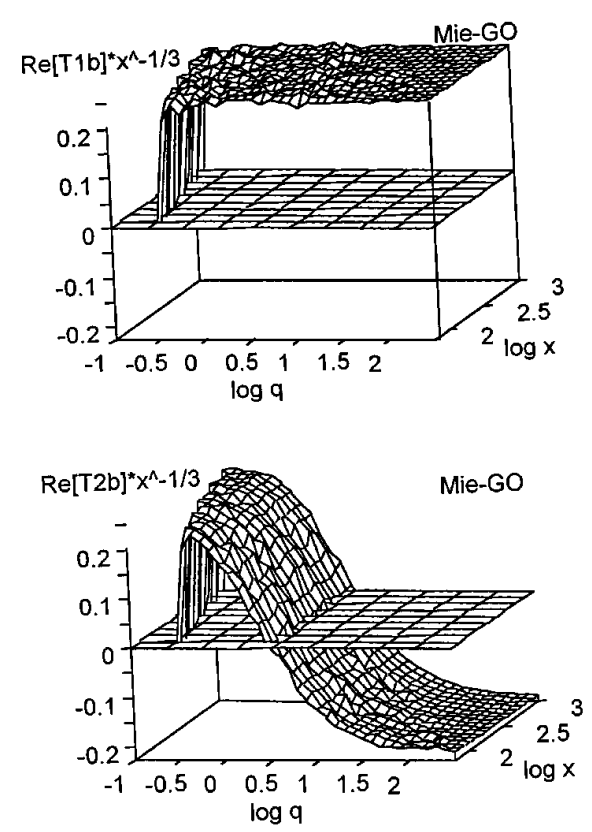

Fig. 7. Real part of edge functions $T 1$ and $T 2$ multiplied by $x^{-1 / 3}$ that was calculated using method b for $0.1<q<316, \operatorname{Im}[m]=0$, and $64<x<1024$. appropriate approximations for scattering amplitudes $S 1$ and $S 2$ (GO, PO, or $\mathrm{AF})$ and the corresponding edge contributions according to Eqs. (13) and (14). In Section 7 we discuss the resulting accuracy for the exact forward direction $(\vartheta=0)$ compared with the accuracy of an approximation that results from the CAM approach. ${ }^{9}$

The calculation time requires for the GO and PO approximations including the edge contribution, using a polynomial fit, is approximately a factor of $x$ lower than the time required to obtain the exact Mie solution. ${ }^{2}$ The calculation time required for the AF depends strongly on the number of terms used for numerical evaluation of Eq. (6) but for the mentioned number of terms is approximately a factor of $x^{1 / 3}$ faster than the exact solution. Only the $64<x<$ 2048 size parameter range and calculation for a specific $x$ value are considered here. For calculation of scattering elements for large enough values of $x$, for which the addition of intensities of different contributions is allowed, we can gain considerably more time using the GO or PO approximation.

\section{Comparison with Complex Angular Momentum Extinction Efficiency}

The approximation we have presented is compared with the CAM approximation presented by Nussenzveig $^{10}$ for the extinction efficiency $Q_{\text {ext }}=4 S(0) / x^{2}$, $64<x<1024$, and $1.1<m<2.5$. For the major part of this $m-x$ area both approximations are equally good..$^{10}$ The CAM approximation is better near $\mu=4$ (Fig. 5) because of an improved $p=1$ contribution. For $\mu<4$ and $q>0.5$ obtained with the $\mathrm{AF}$ and GO methods, respectively, the method we have presented is better. The CAM approximation neglects penetration of outside complex surface waves into the sphere ${ }^{7}$ leading to the condition $|m-1|^{1 / 2} x^{1 / 3}=\mu^{1 / 2} \gg 1$. When the Brewster angle approaches the edge $(q>0.5$, Section 6$)$ transmission into the sphere for polarization 2 is probably not negligible, leading to less accurate CAM results. For smaller values of $x$ the width of the edge domain is larger, so the method presented here leads to worse results.

\section{Summary}

It has been shown that edge functions $T 1$ and $T 2$ as used by van de Hulst ${ }^{1}$ can be determined with a rigorous Mie solution by subtracting appropriate approximations. The relative error of the edge functions determined in this way is approximately $10 \%$ for parameter $x=64$ and decreases as $x^{-2 / 3}$ for higher values of $x$. Adding the edge contributions significantly improves the approximate methods to calculate forward-scattering patterns. The method presented does not give a physical explanation for the resulting edge functions but for some cases leads to a better approximation for the extinction efficiency and probably also for near-forward scattering, 
than an earlier presented expression resulting from the powerful CAM approach. ${ }^{10}$

Appendix A. Nomenclature

$\begin{aligned} a & \text { Sphere radius, } \\ J_{0}, J_{1}, J_{2} & \text { Integer Bessel functions of the first } \\ & \text { kind, } \\ m & \text { Relative refractive index, } \\ n & \text { Refractive index, } \\ p & \text { Ray number, } \\ q & \sqrt{1+m^{2}} x^{-1 / 3} \\ r, r 1, r 2 & \text { Reflection coefficients, } \\ S, S 1, S 2 & \text { Scattering amplitude functions, } \\ T, T 1, T 2 & \text { Edge functions, } \\ x & \text { Size parameter of } 2 \pi a / \lambda, \\ \Delta x & \text { Size parameter range, } \\ \alpha & \text { Angle of incidence, } \\ \lambda & \text { Wavelength, } \\ \mu & (m-1) x^{2 / 3}, \\ \rho & 2(m-1) x, \\ \vartheta & \text { Scattering angle, } \\ \varphi & \text { Azimuth angle, } \\ \tau & \pi / 2-\alpha, \\ \tau_{\text {edg }} & \text { Limit of edge domain, } \\ a, b & \text { Subscripts as calculation methods, Eq. } \\ & (15) \text { or Eq. (16). }\end{aligned}$

\section{References}

1. H. C. van de Hulst, Light Scattering by Small Particles (Wiley, New York, 1957), Chap. 9, p. 114.

2. G. F. Bohren and D. R. Huffman, Absorption and Scattering by Small Particles (Wiley, New York, 1983), Chap. 4, p. 82.

3. D. S. Langley and P. L. Marston, "Forward glory scattering from bubbles," Appl. Opt. 30, 3452-3458 (1991).

4. D. S. Langley and M. J. Morrell, "Rainbow-enhanced forward and backward glory scattering," Appl. Opt. 30, 3459-3467 (1991).

5. Ref. 1, Chap. 12, p. 208.

6. Ref. 1, Chap. 17, p. 349.

7. H. M. Nussenzveig, "High-frequency scattering by a transparent sphere," J. Math. Phys. 10, 82-125 (1969).

8. H. M. Nussenzveig, "Complex angular momentum theory of the rainbow and the glory," J. Opt. Soc. Am. 69, 1068-1079 (1979).

9. H. M. Nussenzveig, Diffraction Effects in Semiclassical Scattering (Cambridge U. Press, London, 1982), Chap. 12, p. 143.

10. Ref. 9, Chap. 13, p. 167.

11. T. W. Chen, "High energy light scattering in the generalized eikonal approximation," Appl. Opt. 28, 4096-4102 (1989).

12. T. W. Chen, "Modified $P-Q$ plot of light scattering for large size parameters," Opt. Lett. 15, 461-462 (1990).

13. S. K. Sharma, "On the validity of the anomalous diffraction approximation," J. Mod. Opt. 39, 2355-2361 (1992).

14. J. Petykiewicz, Wave Optics (Kluwer Academic, Boston, Mass., 1992), Chap. 2, p. 53.

15. Ref. 1, Chap. 7, p. 85 . 